\title{
EDITORIAL
}

For reprint orders, please contact: reprints@futuremedicine.com

\section{When to consider alternatives to front-line immune therapies in metastatic melanoma}

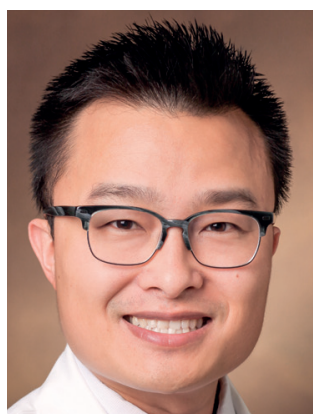

Daniel Y Wang ${ }^{*, 1}$

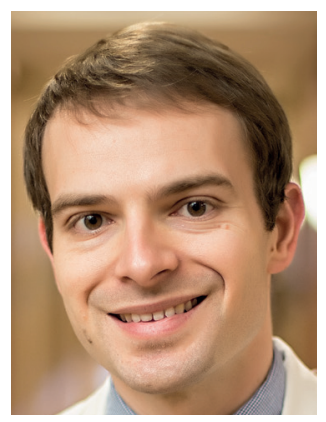

Douglas B Johnson'
“These treatments [immune therapies] are considered in the front-line setting due to the depth and durability of response observed in multiple clinical trials.”

First draft submitted: 10 March 2017; Accepted for publication: 4 April 2017; Published online: 10 May 2017

The introduction of effective therapies in the form of immune-checkpoint inhibitors has dramatically changed the treatment of metastatic melanoma. Prior to this period, metastatic melanoma had a dismal prognosis with a 5 -year overall survival (OS) of less than $10 \%$ [1]. The treatments at the time included high-dose IL-2 and cytotoxic therapies, such as dacarbazine and temozolomide. While the high-dose IL-2 was accompanied by significant toxicities, approximately $6 \%$ of the patients experienced durable remissions [2]. Cytotoxic therapies had even poorer response rates with no proven survival advantage over supportive care; thus, effective therapies were desperately needed. Early studies inhibiting the first checkpoint molecule, CTLA-4, culminated in the development of ipilimumab, the first immune checkpoint inhibitor in melanoma, in 2011 [3-6]. Since then, two PD-1-directed checkpoint inhibitors, nivolumab and pembrolizumab, have been approved for patients with metastatic melanoma. These treatments are considered in the front-line setting due to the depth and durability of response observed in multiple clinical trials. Here, we discuss the benefits of front-line immune therapy and when to consider alternative therapies upfront for metastatic melanoma.

\section{Front-line immune therapies}

A commonly used current paradigm for treatment-naive metastatic melanoma involves deciding a patient's candidacy for either monotherapy with an anti-PD-1 agent (nivolumab or pembrolizumab) or combination immune therapy with nivolumab and ipilimumab. Monotherapy with PD-1 inhibitors have produced response rates in up to $44 \%$ of the patients [7]. Longterm data have also shown the durability of these responses, with 5-year OS of 34\% with nivolumab [8]. Furthermore, monotherapy with PD-1 inhibitors exhibited a fairly tolerable safety profile with $9-16 \%$ of the patients experiencing grade 3-4 adverse events (AEs), with a lower incidence

'Department of Medicine, Vanderbilt University Medical Center, Nashville, TN, USA

*Author for correspondence: Tel.: +615 322 8028; Fax:+615 322 8173; daniel.y.wang@vanderbilt.edu 


\section{EDITORIAL}

\section{"If a patient is not deemed a candidate for front-line immune therapy, a rational approach must be taken to decide the best treatment options."}

of true immune-related AEs [7,9]. Despite these advantages, over half of the patients do not respond to PD-1 monotherapy and subsequent studies have focused on combination immune therapy. The only approved combination immune therapy, nivolumab and ipilimumab, improved the response rate to $58 \%$ but caused higher toxicity as more than half $(55 \%)$ of patients experiencing grade 3-4 immune related AEs [7]. Progressionfree survival is superior with the combination, but OS data are pending.

As both approaches are acceptable first-line treatments in metastatic melanoma, particularly given the lack of OS data, a thoughtful conversation regarding the benefits and risks can inform both clinicians and patients. For patients that are young or have excellent performance status, combination therapy is a reasonable option, as the main concern with combination therapy is manageability of severe immune AEs. However, there is no absolute age or performance score limit to combination therapy. In fact, many clinicians favor combination therapy over monotherapy in BRAF V600E wild-type patients with a significant burden of disease and marginal performance status, where rapid tumor shrinkage may be critical.

\section{Considerations against immune therapy}

However, there are certain situations where immune therapy may not be the optimal choice as the front-line therapy. Patients with significant autoimmune disease potentially at risk for exacerbating their underlying disorder would be one high-risk population. As patients with autoimmune diseases have been excluded from most immune therapy trials, these patients have not been studied prospectively. Retrospective studies have suggested that either PD-1 or CTLA-4 inhibitors are safe and efficacious in many patients with pre-existing autoimmune disorders, with manageable and reversible autoimmune flares or conventional immune-related adverse events [10-12]. Despite this, a careful discussion regarding the risks, benefits and alternative therapies with patients with severe autoimmune disease is required. Another key comorbidity that may limit immune therapy is a history of organ transplant, which may induce graft rejection [12]. Other patient characteristics and comorbidities not directly addressed in prospective trials include age and organ dysfunction. In terms of advanced age, even patients in their 90s may experience clinical responses with manageable side effects [13]. Similarly, patients with baseline cardiac, hepatic or renal dysfunction have been shown to experience clinical responses with tolerable safety profile and infrequent worsening of organ dysfunction [14]. Therefore, most other medical comorbidities would not be contraindications for anti-PD-1 monotherapy.

Another controversial decision point is for patients with $B R A F^{\mathrm{V} 600 \mathrm{E}}$ mutations, as it remains unclear whether front-line targeted or immune therapy is most effective. Particularly, patients with bulky disease may benefit from initial combined BRAF and MEK inhibition. In these situations, there may be life-threatening disease that requires rapid tumor response which cannot be easily achieved with immune therapies. We and others, usually initiate combination BRAF and MEK inhibitor therapy in patients with bulky and symptomatic disease given the rapid tumor reductions often observed. However, this is largely anecdotal with the optimal sequencing of targeted therapy and immune therapy yet to be verified in ongoing randomized trials.

Alternative front-line therapies to immune therapies

If a patient is not deemed a candidate for frontline immune therapy, a rational approach must be taken to decide the best treatment options. These options can involve localized therapy with surgery or radiation if appropriate, targeted therapies based on mutational profile (e.g., BRAF or KIT inhibition), and nontargeted therapies.

For patients with limited burden of metastatic disease, for example, solitary disease in the soft tissue, nodes, first-line surgical metastasectomy leading to complete surgical resection can result in prolonged survival [15]. If a patient only has a small $(\leq 2-3 \mathrm{~cm})$ isolated brain lesion, stereotactic radiosurgery (SRS) has similar efficacy as surgery for local control. Multiple studies have shown that SRS results in a high rate of control of the treated lesion and likely improved survival in a highly selected group of patients [16].

Patients who have more widespread disease should obtain mutational testing to determine the presence of actionable mutations. Clearly, the most clinically actionable is $B R A F \mathrm{~V} 600 \mathrm{E}$, which is mutated in nearly half of the patients. Several studies have demonstrated that combination BRAF/MEK inhibitors produce response rates up to $70 \%$ with median OS of up to 2 years. Two regimens have been improved in this setting: dabrafenib plus trametinib and vemurafenib 
plus cobimetinib. The main disadvantage with targeted therapy is the development of acquired resistance which generally occurs within a year; however, durable responses have been reported [17].

Other targetable mutations that should be assessed include NRAS and c-KIT. NRAS mutations are the second most common mutation in cutaneous melanoma occurring in $15-20 \%$ of the patients. Binimetinib, a MEK inhibitor demonstrated modest improvement in progression-free survival (2.8 vs 1.5 months, HR: 0.62; 95\% CI: 0.47-0.80) over dacarbazine and a response rate of $15 \%$ in NRAS-mutant melanoma patients in the Phase III NEMO trial, although this has not been US FDA-approved to date [18]. KIT mutations are present in 15-20\% of the patients with acral or mucosal melanomas. However, only select patients with activating KIT mutations respond to imatinib, a tyrosine kinase inhibitor. Three Phase II studies have shown tumor responses approximately $25 \%$, especially in KIT mutations involving exon 11 and 13 [19-21]. Interestingly, patients with KIT amplifications did not have favorable responses.

For patients lacking targetable mutations, options become more limited and largely rely on earlier immune therapies that have shown variable efficacy. High-dose IL-2 and adoptive cell transfer therapy have been studied for many years, but are still available at select cancer centers. These therapies may present many of the same obstacles as immune checkpoint inhibitors, however, high-dose IL-2 particularly is limited to patients with excellent performance status due its high morbidity. Retrospective studies have demonstrated a response rate of $16 \%$ with approximately $6 \%$ with prolonged survival over 10 years [2]. By collection, expansion and reintroduction of a patient's existing tumor infiltration lymphocytes, adoptive cell transfer therapy has demonstrated a response rate as high as 53\% in a 'highly select population' [22].

A more recently approved intralesional therapy, talimogene laherparepvec (T-VEC), has shown remarkable tumor responses in patients with unresectable locally advanced and stage IVA disease. T-VEC is an oncolytic viral intralesional therapy consisting of a modified herpes simplex virus type 1 with attenuated viral pathogenicity and the addition of GM-CSF as a chemokine to enhance the immune response. Studies have shown that T-VEC led to a durable response of at least 6 months in $16.3 \%$ of the patients with minimal toxicities [23]. Despite having an excellent local control, systemic response has been seen in patients as well. We frequently use T-VEC in patients with more limited disease who have even very poor performance status. Other intralesional therapies are currently under development as well. Clinical trials should also be strongly encouraged as these allow the possibility of nonapproved targeted therapies for particular mutations and other novel treatment strategies. Other options can include cytotoxic chemotherapy, but are rarely considered in the front-line setting.

\section{Conclusion}

The advent of immune therapy has provided patients with metastatic melanoma with a greater arsenal of therapeutic options. However, there are occasional situations where immune therapy as a front-line option may not be optimal. In these cases, targetable therapy toward appropriate mutations provides the best option for a response. Unfortunately, patients without targetable mutations have more limited options where metastasectomy, radiation, or T-VEC is not indicated. Further studies are needed to define patients that are not optimal candidates for immune therapies and to develop more effective treatment options.

Financial \& competing interests disclosure $D B$ Johnson is on advisory boards for Genoptix and BMS and has received research funding from Incyte. The authors have no other relevant affiliations or financial involvement with any organization or entity with a financial interest in or financial conflict with the subject matter or materials discussed in the manuscript apart from those disclosed.

No writing assistance was utilized in the production of this manuscript.

\section{References}

1 Jemal A, Siegel R, Ward E et al. Cancer statistics, 2008. CA Cancer J. Clin. 58(2), 71-96 (2008).

2 Atkins MB, Lotze MT, Dutcher JP et al. High-dose recombinant interleukin 2 therapy for patients with metastatic melanoma: analysis of 270 patients treated between 1985 and 1993. J. Clin. Oncol. 17(7), 2105-2116 (1999).

3 Krummel MF, Allison JP. CD28 and CTLA-4 have opposing effects on the response of T cells to stimulation. J. Exp. Med. 182(2), 459-465 (1995).
4 Leach DR, Krummel MF, Allison JP. Enhancement of antitumor immunity by CTLA-4 blockade. Science 271(5256), 1734-1736 (1996).

5 Hodi FS, O'day SJ, Mcdermott DF et al. Improved survival with ipilimumab in patients with metastatic melanoma. N. Engl. 


\section{EDITORIAL}

J. Med. 363(8), 711-723 (2010).

6 Robert C, Thomas L, Bondarenko I et al. Ipilimumab plus dacarbazine for previously untreated metastatic melanoma. $N$. Engl. J. Med. 364(26), 2517-2526 (2011).

7 Larkin J, Chiarion-Sileni V, Gonzalez R et al. Combined nivolumab and ipilimumab or monotherapy in untreated melanoma. N. Engl. J. Med. 373(1), 23-34 (2015).

8 Hodi FS, Kluger H, Sznol M et al. Durable, long-term survival in previously treated patients with advanced melanoma (MEL) who received nivolumab (NIVO) monotherapy in a Phase I trial. Presented at: 107th Annual Meeting of the American Association for Cancer Research. New Orleans, LA, USA, 16-20 April 2016.

9 Weber JS, D'angelo SP, Minor D et al. Nivolumab versus chemotherapy in patients with advanced melanoma who progressed after anti-CTLA-4 treatment (CheckMate 037): a randomised, controlled, open-label, Phase III trial. Lancet Oncol. 16(4), 375-384 (2015).

10 Johnson DB, Sullivan RJ, Ott PA et al. Ipilimumab therapy in patients with advanced melanoma and preexisting autoimmune disorders. JAMA Oncol. 2(2), 234-240 (2016).

11 Menzies AM, Johnson DB, Ramanujam S et al. Anti-PD-1 therapy in patients with advanced melanoma and preexisting autoimmune disorders or major toxicity with ipilimumab. Ann. Oncol. 28(2), 368-376 (2016).

12 Johnson DB, Sullivan RJ, Menzies AM. Immune checkpoint inhibitors in challenging populations. Cancer doi:10.1002/cncr.30642 (2017) (Epub ahead of print).

13 Johnpulle RA, Conry RM, Sosman JA, Puzanov I, Johnson DB. Responses to immune checkpoint inhibitors in nonagenarians. Oncoimmunology 5(11), e1234572 (2016).

14 Kanz BA, Pollack MH, Johnpulle R et al. Safety and efficacy of anti-PD-1 in patients with baseline cardiac, renal, or hepatic dysfunction. J. Immunother. Cancer 4, 60 (2016).

15 Sosman JA, Moon J, Tuthill RJ et al. A Phase II trial of complete resection for stage IV melanoma: results of Southwest Oncology Group clinical Trial S9430. Cancer 117(20), 4740-4706 (2011).

$16 \mathrm{Yu}$ C, Chen JC, Apuzzo ML et al. Metastatic melanoma to the brain: prognostic factors after gamma knife radiosurgery. Int. J. Radiat. Oncol. Biol. Phys. 52(5), 1277-1287 (2002).

17 Long GV, Weber JS, Infante JR et al. Overall survival and durable responses in patients with BRAF V600-mutant metastatic melanoma receiving dabrafenib combined with trametinib. J. Clin. Oncol. 34(8), 871-878 (2016).
18 Dummer R, Schadendorf D, Ascierto P et al. Results of NEMO: a Phase III trial of binimetinib (BINI) vs dacarbazine (DTIC) in NRAS-mutant cutaneous melanoma. J. Clin. Oncol. 34(Suppl. 1), Abstract 9500 (2016).

19 Carvajal RD, Antonescu CR, Wolchok JD et al. KIT as a therapeutic target in metastatic melanoma. JAMA 305(22), 2327-2334 (2011).

20 Guo J, Si L, Kong Y et al. Phase II, openlabel, single-arm trial of imatinib mesylate in patients with metastatic melanoma harboring c-Kit mutation or amplification. J. Clin. Oncol. 29(21), 2904-2909 (2011).

21 Hodi FS, Corless CL, Giobbie-Hurder A et al. Imatinib for melanomas harboring mutationally activated or amplified KIT arising on mucosal, acral, and chronically sun-damaged skin. J. Clin. Oncol. 31(26), 3182-3190 (2013).

22 Goff SL, Dudley ME, Citrin DE et al. Randomized, prospective evaluation comparing intensity of lymphodepletion before adoptive transfer of tumor-infiltrating lymphocytes for patients with metastatic melanoma. J. Clin. Oncol. 34(20), 2389-2397 (2016).

23 Andtbacka RH, Kaufman HL, Collichio F et al. Talimogene Laherparepvec improves durable response rate in patients with advanced melanoma. J. Clin. Oncol. 33(25), 2780-2788 (2015). 